\title{
Preventing Signal Degradation During Elastic Matching of Noisy DCE-MR Eye Images
}

\author{
Kishore Mosaliganti ${ }^{1}$, Guang $\mathrm{Jia}^{2}$, Johannes Heverhagen ${ }^{2}$, Raghu Machiraju ${ }^{1}$, \\ Joel Saltz ${ }^{3}$, and Michael Knopp ${ }^{2}$ \\ ${ }^{1}$ Department of Computer Science and Engineering \\ ${ }^{2}$ Radiology \\ ${ }^{3}$ Biomedical Informatics, The Ohio State University, Columbus, OH, USA \\ kishore@bmi.osu.edu
}

\begin{abstract}
Motion during the acquisition of dynamic contrast enhanced MRI can cause model-fitting errors requiring co-registration. Clinical implementations use a pharmacokinetic model to determine lesion parameters from the contrast passage. The input to the model is the timeintensity plot from a region of interest (ROI) covering the lesion extent. Motion correction meanwhile involves interpolation and smoothing operations thereby affecting the time-intensity plots. This paper explores the trade-offs in applying an elastic matching procedure on the lesion detection and proposes enhancements. The method of choice is the 3D realization of the Demon's elastic matching procedure. We validate our enhancements using synthesized deformation of stationary datasets that also serve as ground-truth. The framework is tested on 42 human eye datasets. Hence, we show that motion correction is beneficial in improving the model-fit and yet needs enhancements to correct for the intensity reductions during parameter estimation.
\end{abstract}

\section{Introduction}

The model-based quantitative analysis of contrast agent diffusion in dynamic contrast enhanced MRI (DCE-MRI) allows us to estimate the magnitude and spatial distribution of physiological kinetic parameters such as amplitude (A), redistribution rate constant $\left(k_{e p}\right)$ and the elimination rate constant $\left(k_{e l}\right)$ [1]. The quantification of the temporal intensity signal in the tumor region reflects its angiogenic properties such as the vascular density, arrangement and functional permeability within [2. The input to the model is a sequence of DCE-MR images from which a region-of-interest (ROI) encompassing an entire or portion of the lesion is selected. Quantification involves fitting an appropriate curve to the temporal intensity pattern drawn from the ROI. The modeled parameters can be used to relate to lesion specific physiological concepts.

The challenging aspect in quantification is the positioning of the ROI consistently to cover a lesion across all the epochs. The time required for imaging, non-stationary organs such as the eye, kidney, liver and lung, patient movement, breathing, pain and gravitation effects can cause elastic deformation [2]. Hence, 
model fitting errors arise that alter voxel-to-tissue mapping necessitating the use of multiple ROI's across the temporal scale.

Motion correction has been proposed in the past for the DCE-MRI sequences for improving the parameter estimation by placing a single ROI that is temporally consistent. The choice of using rigid [3, 4, 5, or elastic [6, 7] matching methods have depended on the organ and the deformation inherent in it. While initial research focussed on using conventional metrics (e.g. mutual information 8], correlation ratio [9]) with limited success, efforts were made to incorporate the quantification model into the registration cost function 3. Recently, Buonaccorsi et al. 5 proposed using a simplified tracer model directly into the registration metric to develop a localized, 3D translational registration algorithm focussing on the tumor VOI to obtain better model fits. They assumed that the abdominal tumors were sufficiently rigid in comparison to surrounding tissue. In our case, the low spatial resolution of the eye images (256x256) with high acquisition noise does not provide satisfactory rigid registration results. The tumors in the eye deform elastically due to subject movement, soft tissue and eye ball rotation.

While the need for motion correction has been reported in the literature, the presence of registration artifacts has not been investigated. Co-registration is concomitant with image transformations leading to interpolation of the image field on a grid. Interpolation is akin to a low-pass filtering operation and hence pixel intensities are affected. Reduction in pixel intensities affect the amplitude, rise and wash-in or wash-out gradients of the time-intensity curves and hence the parameters.

In this paper, we explore the role of the Demons registration method [10] in providing a better model-fit. Our research leads us to evaluating the intensity changes caused by smoothing during the registration procedure. Experiments are performed using stationary human eye datasets with induced elastic motion to explore signal degradation. We perform anisotropic smoothing to reduce high frequency noise prior to a high-pass filtering stage. The high-pass filter compensates the smoothing that occurs during registration. Finally, histogram matching of the registered image to the original image is done to match the intensities. The framework is then applied to 42 clinical datasets having complex motion. We show that the pharmacokinetic model provides a better fit to the registered signal patterns as compared to the unregistered case. Moreover, the fit is comparable to the case where manual ROI placement is done across all time-points. We show examples where exact boundaries of the tumor are better identified owing to the improved registration. Hence, we motivate the need for applying motioncorrection with smoothing compensation into the quantification of DCE-MRI.

\section{3D Elastic Co-registration: Thirion's Demons}

Thirion [11,12] introduced the concept of diffusing models to perform image matching, an essential component of this study. Image matching is performed through the movement of a deformable grid through a semi-permeable contour of an object surface in the other image. The method was validated using synthesized 


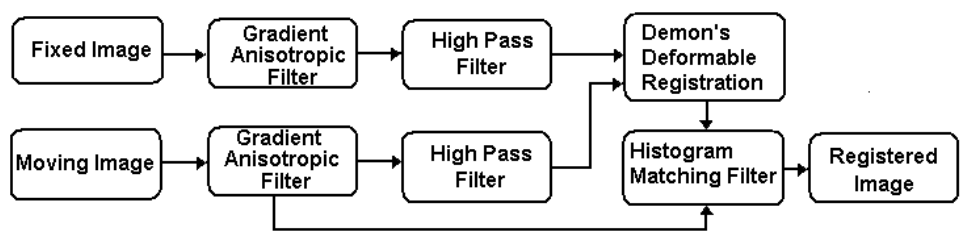

Fig. 1. Deformable registration framework with intensity compensation

deformation on medical images. The method was applied to track heart-motion and 3D matching of inter-patients brain images [13]. In [10], a study of evaluating the temporal variations of lesion volumes for practical applications such as therapeutic intervention effects, therapy monitoring and pharmaceutical trials was conducted. This places our work in perspective. For the DCE-MRI images, each of the epochs provides a $3 D$ volume which is registered to a pre-determined base $3 \mathrm{D}$ volume, chosen at the peak contrast when the features are best defined. The demon's method (see Figure1) was implemented using the National Library of Medicine NLM/NIH's Insight Toolkit (ITK) [14.

A total of 50 iterations yielded satisfactory results with the standard deviation of the Gaussian kernel smoothing the deformation field set at 1.0. The smoothing is essential to avoid inconsistent deformations from occurring. The other components of the framework, namely the gradient-anisotropic filter, high-pass filter and histogram matching filter are explained in the following section.

\section{Validation of Intensity Compensation}

The pharmacokinetic model estimation of the lesion parameters depends on the temporal intensity variation in the ROI encompassing the tumor. Earlier, we mentioned that registration causes smoothing of the image due to the interpolation of the warped image onto a grid. We now seek to understand registration performance based on the changes in pixel intensities. Our task is complicated by the fact that there is no ground truth for pixel intensity validation after registration since a 1-to-1 correspondence with the original image does not exist. Towards this purpose, datasets with no motion in them are selected. Motion is artificially induced in such datasets and then registered. The registered dataset is then compared to the original to study the smoothing effects.

Forty-two patients with eye tumors were included in the study protocol. DCEMRI datasets were acquired on a clinical 1.5-T MR system (GE SIGNA) using a fast gradient-echo sequence (3D-FSPGR). Total scan time per volume was about 8 minutes. After the third phase, a small molecular weight paramagnetic contrast agent (e.g. Gd-DTPA, Magnevist) was injected using a power injector at a constant infusion rate of approximately $18 \mathrm{~s} .3 \mathrm{D}$ volumes of the eye at 28 time-points were acquired, with peak contrast being evident in the $6^{\text {th }}$ epoch.

After manually scanning the forty-two datasets for stationary datasets, EYEOS-P05 and EYE-OS-P28 were selected for this part of the study. We synthesize 


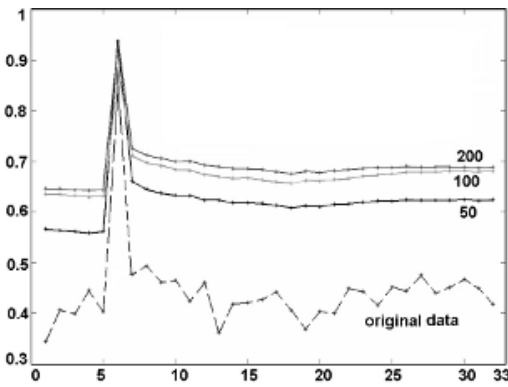

(a)

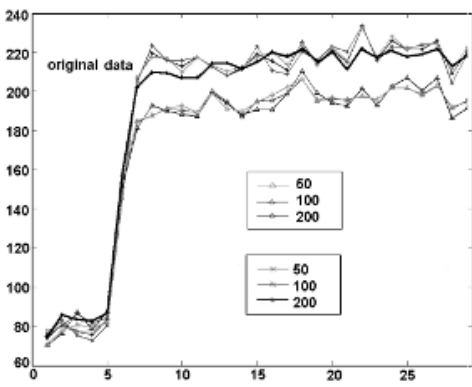

(b)

Fig. 2. (a): Pearson's correlation is plotted for each of the epochs before and after registration. The 6 th epoch is chosen as the base volume causing the occurrence of a peak. (b): The lower curves (triangle markers) show the average ROI intensity in the directly registered volumes. The dark curve represents the true ROI intensity. The upper curves (star markers) represent the average ROI in registered volumes using intensity compensating high-pass filtering.

elastic motion in the 3D volumes by evaluating the displacement of each voxel as a Gaussian function of 4 independent parameters namely, 1. time-point $(4,10)$, 2 . slice location within the volume $(8,10), 3$. rotation angle $\left(10^{\circ}, 50\right)$ about the eye-center and 4. constant translation of 20 vertically. The values in the brackets specify the mean and standard deviation of the independent parameters. The rationale behind this procedure is that deformation is smooth and continuous spatially and relatively faster across the time-points. Further, the deformation in each time-point is maximum at a given slice and gradually declines for the neighboring slices. The translation may be due to blood flow or regular breathing and cannot be correlated across time-points and hence represented as a constant. The Gaussian parameters were chosen iteratively by a oncologist to reflect deformation found in practice. The deformed dataset is interpolated back onto a grid to represent a dataset with motion. The attached video sequences static.avi and deform.avi show the deformation obtained in a particular slice across all time-points. Using the original and deformed volumes, we can now evaluate the registration performance.

Figure 2(a) shows the Pearson's correlation plots of the eye dataset. The bottom (broken) curve indicates the correlation with the base volume ( $6^{\text {th }}$ epoch) prior to registration. The correlation in the volumes was 0.45 prior and 0.7 postregistration on average. Registration improves the correlation significantly. The low values are partly due to the motion, white noise in the images and also from the continuous uptake and washout of the contrast medium. The higher curves indicate the correlation values for 50, 100 and 200 iterations of the registration algorithm respectively. Longer iterative times tend to produce better registrations with diminishing returns progressively.

Figure 2(b) shows that as the registration accuracy improves (as a result of increased iteration), the drop in the average ROI intensity is more pronounced. The three lower curves (triangle markers) are directly registered volumes with- 


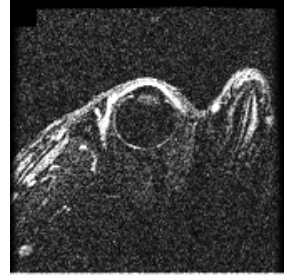

(a)

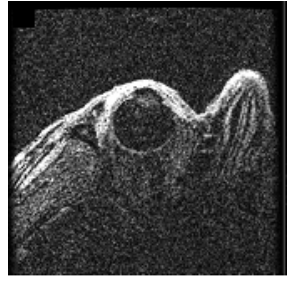

(b)

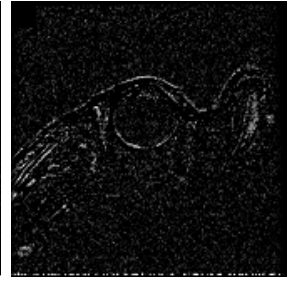

(c)

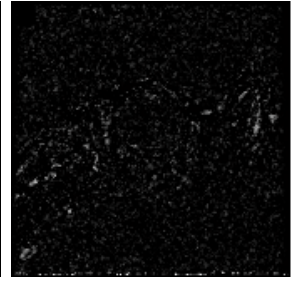

(d)

Fig. 3. (a): Static slice. (b): Motion-affected slice. (c): Difference image. (d): Difference image after registration.

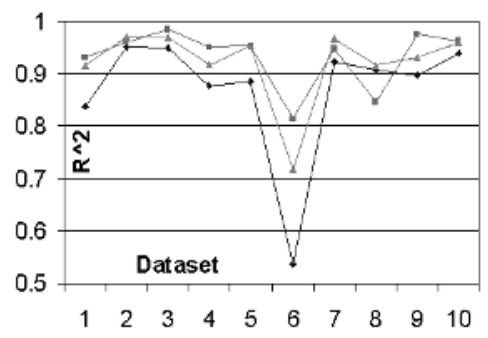

(a)

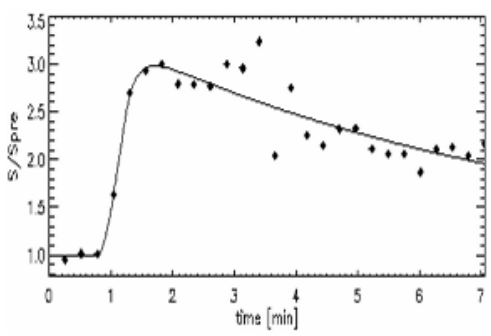

(c)

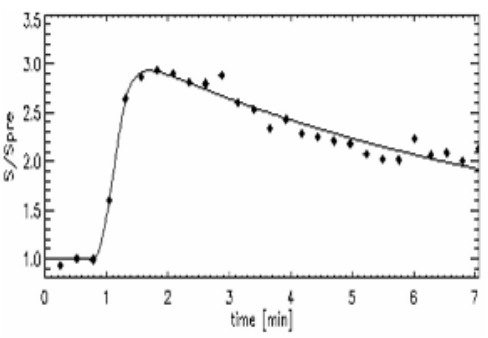

(b)

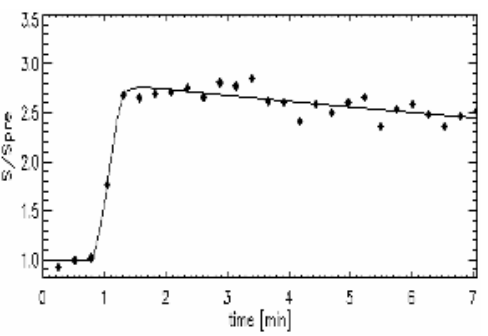

(d)

Fig. 4. (a): $R^{2}$ fit ratios using the pharmacokinetic model for the 3 cases in EYE-OSP06 (8th slice). Fit observed using (b): multiple ROIs in the unregistered (c) single ROI in the unregistered and (d): single ROI in the new registered framework.

out any enhancements for 50, 100 and 200 iterations. In order to counter the smoothing during registration, we perform high pass filtering on the images prior to registration. However, this amplifies the shot noise to a great extent thereby defeating the purpose. Hence, the high-pass filter is preceded by a gradient-based anisotropic diffusion filter [15] that reduces the high frequency noise. Finally, we use a histogram matching procedure after the registration is complete to map the intensities back to the original image as shown in Figure 1. Here, the reference image is the image prior to registration. The histogram matching is done only in a region local to the ROI. We also threshold using mean intensity value in order to eliminate the background voxels from interfering. Using this new framework, 
the registered volumes were scaled as shown by the curves with star markers in Figure 2. These curves now lie in the vicinity of the dark curve that represents the true ROI intensity and share the same trends.

A total of 4 iterations yielded satisfactory results with a time-step of 0.125 and the conductance term set to 1.0 for the gradient anisotropic filter. The high pass filter was implemented as a inverted 2D Gaussian filter with $\sigma=$ 0.75. The normalized frequency domain representation is given by: $F\left(\omega_{x}, \omega_{y}\right)=$ $N\left(1-\frac{1}{\sqrt{2 \pi} \sigma} e^{-\frac{\omega_{x}^{2}+\omega_{y}^{2}}{\sigma^{2}}}\right)$, where $N$ is a normalizing factor to make the Gain $=1$. Figure $3(\mathrm{a}, \mathrm{b})$ shows two slices at the $6^{\text {th }}$ and $10^{\text {th }}$ time-points of the EYEOS-P05 dataset. Slice 3(b) suffers from motion-induced warping. Figure 3(c) is the difference image of 3 (a) and 3(b), showing the mis-alignment. The new difference image after registration is shown in Figure 3(d). The improvements and alignment of the boundaries are easy to observe over 3(c).

Why is filtering important? The crucial importance of DCE-MRI has been in understanding the heterogeneity in the tumor with respect to its vascular arrangement and density. Smoothing of images can potentially be dangerous since it causes the lesion to exhibit a homogenous texture. Preserving this important aspect requires intensity compensation by means of high-pass filtering and without amplifying the noise.

\section{Experiments and Discussion}

In this section, we strive to validate the performance of the registration in terms of the fit provided by the pharmacokinetic model. Three scenarios were considered: 1.) A single ROI was placed over the lesion for a given slice location where the tumor was well-defined and replicated at all other time points in the unregistered volumes. 2.) A trained pathologist placed ROIs for a given slice location consistently for all the time-points in the unregistered volumes. In other words, the ROI was shifted based on observed deformation. 3.) A single ROI was placed over the lesion at a given slice location and replicated at all other time points in the registered dataset. Here, there is no movement of the ROI.

The first case represents the assumption that there is no motion at all. The second situation realizes that there is motion and we manually correct the ROI placement. The third case assumes that there is motion and we use motioncorrection procedures and then place a single ROI. We can therefore expect that the pharmacokinetic model would provide the best fit to the second case and the worst fit to the first case. We try to evaluate the fit that the third case provides.

Goodness of fit Statistics: The fit provided by the model to the experimental data obtained from the three cases was evaluated using the parameter $R^{2}=$ $1-\frac{S S_{\text {error }}}{S S_{\text {mean }}}$, where $S S_{\text {error }}$ is the sum of the squares of the distance between the experimental data and the best-fit curve and $S S_{\text {mean }}$ is the sum of squares from the mean of all points [16]. When $R=0$, the fit provided is no better than the mean horizontal line. When $R=1$, the curve fits the points exactly. 


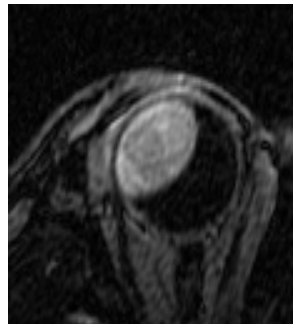

(a)

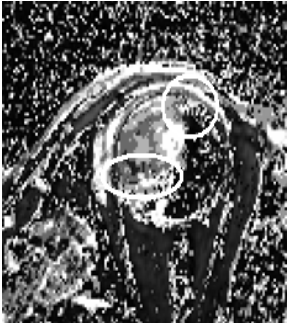

(b)

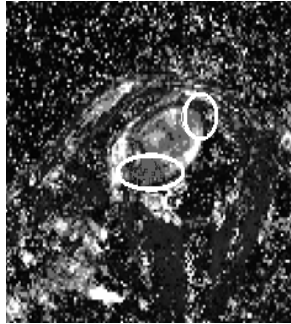

(c)

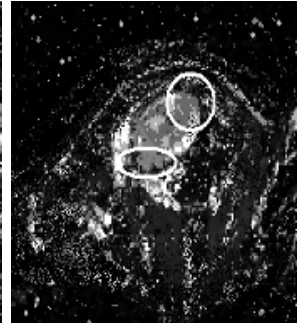

(d)

Fig. 5. (a): EYE-OS-P25 with tumor (b): Unregistered (c): Registered dataset without enhancement (d) Registered dataset with enhancement

Analysis: We picked 10 datasets randomly and plot the $R^{2}$ fit obtained for the 3 cases in Figure 4 (a). As expected, the fit obtained from case 2 (square markers) is better than case 1 (diamond markers). Case 3 (triangle markers) performed better than the case 1 and matched or outperformed case 2 (the ideal case). Hence, the registered dataset with a single ROI consistently encompassed the tumor region for the given pharmacokinetic model. An example of the fits for the EYE-OS-P06 is plotted for illustration. The model fits (Figure $4 \mathrm{~d}$ - case 3 ; 4 $\mathrm{b}$ - case 2) better than (4. - case 1).

Pixel-mapped model: While the above experiments were based on average signal intensities drawn from an ROI, we now investigate the results on applying the model to each voxel in the $3 D$ volume. The two important lesion parameters $\left(\mathrm{A}\right.$ and $k_{e p}$ ) are intensity-mapped and plotted. The noisy pixelwise analysis shows that the lesion has intense local enhancement with spot noise. Figure 5 shows the pixel mappings for the tumor in EYE-OS-P25 dataset. We make three observations: 1) In the unregistered dataset, the tissue structures such as the upper rim of the eye are more well-defined in terms of the enhancement locally. The structures are however absent in the directly registered case. The reason for this is the smoothing that happens in registration destroys the thin structures and reduces the enhancement pattern per pixel. 2) The registered datasets $(\mathrm{c}, \mathrm{d})$ define the tumor boundaries better than the unregistered case especially in the upper and lower poles (white circles). It indicates that there are more pixels that are enhanced indicating a better fit to the model. 3) The registered dataset with the high pass filtering improves over the directly registered case by reducing the shot noise, mapping the other peripheral eye structures and providing a better characterization of the tumor interior.

In conclusion, the effect of blurring in the pixel intensities and on the lesion parameters is explored. We incorporate filtering operations prior to registration to reduce noise and pre-empt signal degradation. Our validation covers stationary datasets with induced motion for ground-truth. The framework is tested on clinical cases. We found that motion artificially changes lesion morphology near the boundaries. Using our new framework, the effect for contrasting differences in the enhancement pattern for quantitative evaluation was pronounced. 


\section{References}

1. Taylor, J.S., Tofts, P.S., Port, R., et al: MR imaging of tumor microcirculation: Promise for the new millennium. J. Magn. Reson. Imaging 10 (1999) 903-907

2. Knopp, M.V., Weiss, E., Sinn, H.P., et al: Pathophysiologic basis of contrast enhancement in breast tumors. J. Magn. Reso. Imaging 10 (1999) 260-266

3. Hayton, P., Brady, M., Tarassenkoa, L., et al: Analysis of dynamic MR breast images using a model of contrast enhancement. Med. Image Anal. 1 (1997) 207224

4. Bidaut, L.M., Vallee, J.P.: Automated registration of dynamic MR images for the quantification of myocardial perfusion. J. Magn. Reson. Imaging 13 (2001) 648-655

5. Buonaccorsi, G.A., Roberts, C., Cheung, S., et al: Tracer kinetic model-driven registration for dynamic contrast enhanced MRI time series. In: MICCAI'05, Lect. Notes in Comp. Sc. (2005)

6. Lucht, R., Knopp, M.V., Brix, G.: Elastic matching of dynamic MR mammographic images. Magn. Reson. in Medicine 43 (2000) 9-16

7. Rueckert, D., Sonoda, L., Hayes, C., et al: Nonrigid registration using free-form deformations: application to breast MR images. IEEE Trans. Med. Imag. 19 (1999) $712-721$

8. Maes, F., Vandermeulen, D., Suetens, P.: Medical image registration using mutual information. Proceedings of the IEEE 91(10) (1996) 1699-1722

9. Roche, A., Malandain, G., Pennec, X., et al: The correlation ratio as a new similarity measure for multimodal image registration. Lect. Notes Comput. Sc. 1496 (1998) 1115-1124

10. Thirion, J.P., Calmon, G.: Measuring lesion growth from 3D medical images. In: Nonrigid and Articulated Motion Workshop (NAM'97). (1997)

11. Thirion, J.P.: Fast non-rigid matching of 3D medical images. In: Medical Robotics and computer aided surgery, MRCAS'95. (1995) 47-54

12. Thirion, J.P.: Fast non-rigid matching of 3D medical images. In: Computer Vision and Pattern Recognition, CVPR'96. (1996)

13. Thirion, J.P., Subsol, G., Dean, D.: Cross validation of three inter-patients matching methods. In: Visualization in Biomedical Computing VBC'96, Lect. Notes in Comp. Sc. Volume 1131. (1996) 327-336

14. Ibáñez, L., Schroeder, W.: The ITK Software Guide. The Insight and Registration Toolkit [www.itk.org], Kitware, Inc. (2003)

15. Perona, P., Malik, J.: Scale-space and edge detection using anisotropic diffusion. IEEE Trans. on Pattern Anal. and Mach. Intell. 12 (1990) 629-639

16. Fan, X., Medved, M., River, J.N., et al: New model for analysis of dynamic contrastenhanced mri data distinguishes metastatic from nonmetastatic transplanted rodent prostrate tumors. Magn. Reson. in Med. 51 (2004) 487-494 